\author{
Enzo Caffarelli \\ Laboratorio Internazionale di Onomastica, \\ Università di Roma "Tor Vergata"
}

\title{
"Piazza Bologna" a Roma: studio di caso di un panorama onimico. Processi transonimici di macro- e microtoponimi
}

Che fine fanno i toponimi è una domanda che può apparire curiosa solo a chi non abbia piena percezione del continuo riciclaggio cui i nomi di luogo sono sottoposti, passando da una tipologia (o categoria) all'altra in àmbito onimico, oppure lessicalizzandosi. In questo contributo si cercherà: a) di analizzare un corpus di toponimi d'ogni genere raccolti in uno specifico àmbito spaziale e temporale, una piazza della città di Roma, Piazza Bologna, e i suoi dintorni, nel pomeriggio del 2 e nella mattinata del 25 ottobre 2014 ; b) di esaminare il processo transonimico che tali toponimi hanno subito; e c) di verificare se esistono percorsi transonimici ricorrenti o più tipici di particolari tipologie di toponimi.

\section{Premesse terminologiche e metodologiche}

Alcune premesse terminologiche sono necessarie. In àmbito linguistico non italiano, ma soprattutto inglese, è stato introdotto in tempi relativamente recenti il concetto noto internazionalmente come "linguistic landscape" (LL), con l'annessa idea del "nome proprio nel panorama linguistico", che risale almeno a Landry, Bourhis (1997): «The language of public road signs, advertising billboards, street names, commercial shop signs, and public signs on government buildings combines to form the linguistic landscape of a given territory, region, or urban agglomeration». A tale concetto si può affiancare quello analogo e quasi iponimico di "onomastic (meglio: onymic) landscape", ossia la raccolta e analisi di quanti e quali nomi propri o voci di lessico con quote di proprialità - partendo dall'ipotesi, non da tutti considerata o condivisa, che sia impraticabile una differenziazione tra nomi propri e lessico netta e definita una volta per sempre in diacronia, diatopia, diastratia, diafasia e diamesia - siano presenti in un dato "linguistic landscape" [Puzey 2011; Caffarelli 2014a]. 
Quanto ai concetti di macro- e microtoponimo, le ambiguità di una ripartizione sicura, nella quale all'uno e all'altro termine corrispondano caratteristiche costanti in termini formali e designativi, sono riflesse dalle varie motivazioni e modalità con le quali la comunità scientifica ricorre all'uso di tali definizioni. In una rapida inchiesta condotta tra colleghi sul significato di micro- e macrotoponimo ho raccolto nel 2012 definizioni alquanto differenziate [Caffarelli 2013a]; in particolare il concetto di +abitato vs. -abitato, ma anche quello di +grande vs. -grande sono soggetti a pareri diversi e a volte decisamente contrastanti tra loro; del resto, in un volume di questa stessa collana, Paolo Poccetti (2014) ha lucidamente inquadrato tutte le difficoltà.

A tutto ciò aggiungo una perplessità che a me pare irrisolvibile con gli strumenti terminologici attuali: tra il macro- e il micro- devono pur esistere una o più categorie intermedie che raccolgano tutto ciò che non è né "grande" né "piccolo" e che in lingua italiana viene rappresentato tout-court come toponimo, voce che viene dunque ad essere una subtipologia di "toponimo", e dunque iponimo e iperonimo di sé stesso, il che non è accettabile né perspicuo (al contrario, mi pare assodato e accettabile il termine coronimo indicante un territorio più vasto di quello individuato dal toponimo, per macro che sia).

Con odonimo s'intende qui qualsiasi tipologia di area di circolazione comprese le piazze per le quali alcuni utilizzano la voce plateonimo (o ingl. agoronym, «A type of urbanonym, naming a town, city square, or marketplace» [vedi Room 1996: 4]).

A proposito di odonimi di una certa rilevanza, ricorro al termine iperonimo odonimico, per segnalare, sulla scia degli studi di Nikitin (2011) sull'odonimia di alcune città italiane quel nome di via o di piazza che, per i motivi più vari, viene ad assumere convenzionalmente un valore denominativo molto più ampio del suo referente originale, inglobando i vari odonimi che vi si trovano intorno, sempre tenendo conto delle differenze tra odonimia ufficiale e colloquiale e delle mappe mentali disegnate da ogni persona [vedi anche Lucantoni, Porcaro, Vigliarolo 2009].

Per transonimia, transonimico e transonimizzazione intendo ciò che attiene a qualsiasi passaggio di un nome da una tipologia (o subtipologia) all'altra all'interno dell'onimia; tale passaggio può essere correlato o irrelato, a seconda che la motivazione del cambiamento abbia un legame con il referente oppure sia casuale e fungibile, rifletta cioè nel secondo caso, com'è tipico dei repertori odonimici dei centri urbani moderni, un criterio classificatorio (celebrativo-commemorativo o semplicemente enumerativo che sia). La correlazione può essere storica, se dovuta a un qualsiasi evento che ha posto in relazione l'eponimo con l'omonimo derivato, oppure metaforica o anche metonimica.

Una transonimizzazione può definirsi lineare se presuppone uno o più cambiamenti progressivi di tipologie (considerando tra queste anche la lessicalizzazione); bidirezionale se il percorso comprende uno o più ritorni sulla tipologia di partenza (o su una tipologia di transito del percorso); duplice, triplice, ecc. se si caratterizza come più itinerari originati dal nome proprio di partenza o attraverso 
biforcazioni lungo il percorso; stellare o nucleare se dalla voce iniziale si originano vari transonimi ciascuno indipendentemente dall'altro. Ogni tratto del percorso può essere diretto, qualora se ne consideri una sola tappa (da $x$ a $y$ di tipologia differente), mediato quando tra il punto $x$ e il punto $y$ (che possono rappresentare tipologie sia identiche sia differenti) esiste un punto $z$ che, appunto, media il passaggio e lo rende bifasico.

Infine, accolgo in pieno la distinzione ormai assodata in àmbito slavistico e in gran parte in àmbito germanistico (ma ancora raramente e timidamente in quello romanistico) tra onimia e onomastica e tra il gruppo di termini in -onimia e in -onomastica, considerando i primi come il corpus (e dunque la lettura, la raccolta, l'indicizzazione, ecc. dei nomi) e i secondi come ogni tipo di analisi, classificazione, elaborazione, di quegli stessi nomi.

Nel descrivere lo studio di caso annunciato, mi permetto di ricorrere a uno stile narrativo personalizzato che spero consenta al lettore di seguire l'osservazione dell' "onymic [togli spazio] landscape". Il lavoro è consistito nell'osservazione diretta - nella Piazza Bologna di Roma e nelle aree di circolazione vicine - di tutti i nomi propri toponimici o detoponimici che apparivano leggibili, senza distinzione di grandezza, medium, funzione, unicità (per es. un odonimo) o replicabilità (per es. un crematonimo inteso come prodotto commmerciale), né del carattere pubblico o privato (categorie tra le quali il discrimine è peraltro incerto e discutibile): una sorta di namewatching non troppo lontano da metodologie di osservazione ben più note, come il birdwatching. I dati, trascritti su un taccuino, sono stati completati dalla ricerca in rete che ha permesso di cogliere alcune peculiarità minute che le insegne e quanto disponibile a vista non contenevano.

\section{Stazioni delle linee metropolitane e iperonimi odonimici}

Piazza Bologna - odonimo istituito con delibera del Comune di Roma del 21 luglio 1920 - si trova nel grande quartiere Nomentano (il V), al cui interno si hanno suddivisioni di carattere non ufficiale e tra queste spicca il Quartiere Italia o delle Province, dove si colloca la piazza esaminata. Per raggiungerla dal mio studio sito presso Viale Libia, il percorso è servito dalla linea B1 della metropolitana, che presenta una stazione in entrambi i siti (Libia e Bologna).

Come d'uso in altre città, una stazione dei "treni metropolitani" (di qui: "metropolitana") viene definita semplicemente attraverso il suo denotante senza denotato, attraverso una triplice ellissi (con effetto metonimico): stazione della metropolitana di Piazza Bologna > stazione di Piazza Bologna > stazione Bologna $>$ Bologna; anche il display nelle carrozze del treno segnala "prossima fermata Bologna", mentre il nastro audio preregistrato annuncia ellitticamente "in arrivo a Bologna" e tutte le insegne-cartello all'interno e all'esterno della stazione registrano semplicemente "Bologna". 
Il percorso si trasforma pertanto nell'espressione "da Libia a Bologna". Poiché in italiano i nomi di città non sono normalmente preceduti dall'articolo (eccezion fatta, s'intende, per le forme in cui l'articolo determinativo è parte integrante del toponimo: La Spezia, La Maddalena, Il Cairo, L'Aja...), l'espressione "andare da Libia a Bologna" non dovrebbe confondersi con un viaggio dallo Stato nordafricano al capoluogo della regione Emilia-Romagna, che suonerebbe "andare dalla Libia a Bologna". Tuttavia la totale identificazione formale tra Bologna città italiana e Bologna stazione della metropolitana romana, se decontestualizzata, mantiene una sua forte ambiguità: "scendo sempre a Bologna", "c'è un ristorante proprio vicino a Bologna", "ho affittato casa a Bologna" potrebbero indicare tanto il macrotoponimo emiliano quanto il microtoponimo romano ${ }^{1}$.

Le stazioni della linea B della metropolitana di Roma (il cui secondo ramo, denominato B1 e comprendente la stazione Libia, è stato inaugurato nel 2012) sono ovviamente riprodotte nelle tabelle affisse all'interno delle carrozze dei treni e dappertutto sulle pareti delle stazioni. A "Bologna" si leggono dunque altri nomi-stazione, alcuni tratti da odonimi deantroponimici, altri da microtoponimi non strettamente odonimici come invece Libia o Bologna (o per i quali l'esistenza di un odonimo correlato è secondario): è il caso almeno di Termini (la stazione centrale), Policlinico (il complesso ospedaliero intitolato al re Umberto I), Castro Pretorio (la sede della guardia pretoriana in epoca romana), Colosseo e Circo Massimo (i teatronimi più noti dell'antica Roma), Piramide (la tomba di Caio Cestio a forma di piccola piramide), Ostiense e Garbatella (due grandi quartieri di Roma sud), Basilica di San Paolo (una delle basiliche maggiori della capitale italiana), Eur Palasport (il palazzo dello sport nel quartiere Eur, acronimo per Esposizione Universale Roma). In questi casi, dunque, il processo transonimico è diretto e non mediato e si presenta correlato (la stazione si chiama Colosseo perché costruita in prossimità del Colosseo); invece nei casi mediati, che qui hanno subìto un processo transonimico in due tappe, si rileva una fase irrelata (la piazza è dedicata a Bologna senza alcuna motivazione specifica) e una fase correlata (la stazione è denominata Bologna perché si trova nei sotterranei di Piazza Bologna). La linea B, dopo la biforcazione nella B1, tocca tra le altre la stazione Monti Tiburtini: qui il processo transonimico, a ritroso nel tempo, conduce all'identico odonimo romano e questo all'oronimo che a sua volta deriva da un econimo: Tibur, antico nome dell'odierna Tivoli in provincia di Roma; Tivoli, poi, ha avviato un ulteriore e ben noto percorso transonimico: grazie infatti alle bellezze rinascimentali della Villa d'Este con sue scenografiche fontane e giochi d'acqua, ha

${ }^{1}$ Lo stesso vale per ogni altro toponimo trasformato in stazione metropolitana: si veda ad esempio, nel caso di Roma, Cipro, Lepanto, Monti Tiburtini, Spagna, ecc.; o nel caso di Milano, dove la rete è più ricca e il numero di stazioni ben più elevato che a Roma: Bisceglie, Brenta, Caiazzo, Domodossola, Famagosta, Istria, Lima, Loreto, Marche, Palestro, Sondrio, Udine, Uruguay, Zara, solo per citarne alcune. 
ispirato il parco parigino Jardin de Tivoli (1766-1842), a sua volta preso a modello (insieme ai Vauxhall Gardens londinesi) per i Tivoli Gardens di Copenaghen².

Tornando al repertorio odonimico di Roma legato al toponimo Bologna, andrà notato in parentesi che figurano anche Vicolo del Bologna (artigiano cinquecentesco), Via Bolognano (comune dell'Abruzzo), Via Bolognetta (comune della Sicilia), Via Bolognola (comune delle Marche) e Largo Castel Bolognese (comune della Romagna), per i quali si è avuto il passaggio da toponimo a odonimo ${ }^{3}$; Via Giovanni da Castel Bolognese (incisore di medaglie del sec. XVI) con passaggio mediato dalla collocazione in una catena antroponimica; e inoltre Via Franco Bolognese (miniaturista del sec. XVII), Via Francesco Bolognesi (architetto coevo del precedente), Via Alberto Bolognetti (cardinale del sec. XVI), Via Giorgio Bolognetti (benefattore) e Via Lodovico Bolognini (giureconsulto del sec. XV), casi in cui un cognome di chiara derivazione toponimica si è fatto odonimo ${ }^{4}$.

Come si accennava a proposito degli iperonimi odonimici, Bologna (intesa come piazza) ancor prima della costruzione della stazione della metropolitana, relativamente recente (1990), aveva assunto un valore locativo più ampio: con l'espressione "in zona Piazza Bologna" s'intendeva e s'intende una vasta area di riferimento compresa pressappoco tra la via Nomentana, la Circonvallazione Nomentana, la Via Tiburtina e il Viale Regina Margherita. Tale iperonimo dopo il 1990 si è via via rafforzato in popolarità, nello stesso tempo riducendosi a "zona Bologna" (o ancor più ellitticamente "Bologna"), a motivo della semplificazione nell'oralità a ciò che è strettamente necessario per la comprensione tra parlanti.

A dimostrazione di tale argomento, spicca l'esempio dell' Hotel Mercure Roma Piazza Bologna, in cui il nome della piazza è parte integrante della denominazio-

${ }^{2}$ Voluti nel 1843 dal re Cristiano VII, si tratta di un parco divertimenti che ispirò perfino i creatori di Disneyland (vi s'incontrano la Tivoli Guard, banda musicale di ragazzi in uniforme, il Tivoli Concert Hall, il Tivoli Congress Center, il Tivoli Aquarium...); l'importanza del parco danese è alla base di ulteriori identiche denominazioni per vari parchi a tema presenti nel mondo, dalla Spagna alla Slovenia al Giappone. Tale vocazione al "divertimento" legata al nome Tivoli ha fatto sì che numerosi teatri nel mondo si richiamassero al toponimo: incontriamo così un Tivoli Theatre a Washington, uno a Chicago e uno a St. Louis negli USA, uno ad Aberdeen in Scozia e uno in Australia (creato nel 1911 e demolito nel 1969); inoltre lo stadio di Aachen, in Germania, si chiama New Tivoli (il precedente stadio è conosciuto come Old Tivoli), e con l'appellativo di Tivoli-Neu si denomina lo stadio di Innsbruck, in Austria. Un'ulteriore spinta alla popolarità di Tivoli data 12 settembre 2008, quando uno scalatore tiburtino, Cesare Giuliani, ha raggiunto per primo una cima inviolata dell'Himalaya e l'ha battezzata Tivoli Peak.

${ }^{3}$ Si tenga conto che Bolognola vale etimologicamente 'piccola Bologna', mentre Bolognetta si lega a un antroponimo detoponimico e Bolognano appare invece una rietimologizzazione da un personale Velonius o simile.

${ }^{4} \mathrm{Si}$ può aggiungere che dal toponimo Bologna derivano una ventina di diversi cognomi italiani: Bologna, Bolognani, Bolognari, Bolognase*, Bolognati/-ato, Bologne*, Bolognese/-i, Bolognetta*, Bolognetti, Bologni, Bolognin/-ni/-no, Bolognisi*, Bolognone/-i; rarissimi e frutto di errate trascrizioni i tipi contrassegnati da un asterisco posposto; non esistono invece con Bologna cognomi sintagmatici, accompagnati da articoli o preposizioni. 
ne (l'odonimo si trasforma dunque in crematonimo con funzione indicativa-locativa) ma che si trova in Via Reggio Calabria; analogamente in via Berengario ha sede il Centro Veterinario Piazza Bologna; sulla Circonvallazione Nomentana il punto vendita e riparazione pneumatici Bologna Pneus; in Via Piccarda Donati il garage Bologna; in Via Lorenzo il Magnifico il garage Bologna 2, la scuola di lingue The New British Center Piazza Bologna srl e le agenzie immobiliari Bologna srl e Studio Bologna 1; in Via Catania l'agenzia Bologna Immobiliare srl; in via Catanzaro le altre agenzie immobiliari Bologna Università e Toscano-Bologna Università; in Via Cremona la Buildingcase Bologna Immobiliare; in Via Apuania la Clinica Iphone Piazza Bologna (forniture per uffici); in Via Ernesto Monaci il Bologna Phone Center SAS; in Viale XXI Aprile, il Laboratorio analisi cliniche Bologna e la libreria Feltrinelli Point Piazza Bologna; solo alcune di queste vie afferiscono nella Piazza Bologna, dove incontriamo la Farmacia Piazza Bologna, la Tabaccheria Bologna, il Bar Bologna, e il B\&B Bologna; le altre si trovano a una certa distanza e la loro denominazione, specie dove il denotante Bologna è accompagnato dal denotato Piazza, possono essere fonte di fraintendimento. In Via Stamira esisteva inoltre il Cinema Bologna progettato nel 1949 (poi divenuto Academy Hall e dopo il 2000 trasformato in sala bingo).

Tutto ciò non impedisce, evidentemente, che anche le aree di circolazione comprese nella cosiddetta zona Bologna presentino insegne commerciali che propongono il proprio nome; in Viale Ippocrate per es. incontriamo la drogheria $\mathrm{Ca}$ stroni a Viale Ippocrate, l'Ippocrate Service Management, l'agenzia immobiliare Studio Ippocrate il Laboratorio Ippocrate di analisi cliniche e lo Studio Medico Ippocrate (gli ultimi due particolarmente appropriati, richiamando il nome del più celebre medico dell'antichità); in Via Lorenzo il Magnifico la Farmacia Il Magnifico e la Residenza il Magnifico; in Viale XXI Aprile l'Agenzia autoscuola XXI Aprile e la Unicredit Banca-Roma XXI Aprile; in Via dei Foscari la Foscari House Bed \& Breakfast; in Via Catania la drogheria Castroni a Via Catania (denominazione di cui l'odonimo è divenuto parte integrante, dato che si trova un'identica insegna in Viale Ippocrate per un'enoteca), la Frutteria Catania SAS e 'Mpare Bar Catanese (che crea una curiosa ambiguità sul significato dell'aggettivo: etnico o deodonimico? $)^{5}$; in Viale delle Province 1'Hotel delle Province, il Caffè Provincie e il cinematografo delle Provincie; e così via.

Significativa è la presenza dell'Autorimessa Parking delle Provincie e del Punto Immobiliare Provincie non nel viale o nel piazzale omonimo, ma nella vicina Via della Lega Lombarda; ciò rappresenta, infatti, una spia seppur tenue dell'ulteriore suddivisione iperonimica dell'area (Province come iponimo di Bologna ma iperonimo di Lega Lombarda e altri odonimi), se non di una separazione dall'iperonimo principale.

${ }^{5}$ Può derivare infatti sia dalla caratterizzazione dei prodotti offerti o dalla provenienza siciliana (da Catania in particolare) del gestori, sia banalmente dal nome della strada. 
Nikitin (2011) ha esemplificato per il centro storico di Roma i confini degli iperonimi odonimici, dove cioè l'uno perde consistenza e popolarità a favore dell'altro che subentra. Andrà anche notato che un esercizio commerciale può recare nell' insegna un odonimo differente da quello su cui insiste ma di pari grado (non iperonimico): è il caso, nell' area qui considerata, della farmacia Farmacrimi Apuania, non in Via Apuania ma in Via Michele di Lando; e il negozio di calzature Ravenna srl non in Via Ravenna bensì in Via Livorno.

Numerose, in questo caso dalla ricerca in rete, risultano inoltre formule del tipo "pasticceria Roma Piazza Bologna - Pane al Pane" o "Pizzeria Roma Piazza Bologna - Pizza dei Cinque", dove soltanto la seconda parte della scrizione (corsivo nostro) corrisponde all'insegna ufficiale dell'esercizio commerciale, mentre la prima segnala la tipologia del locale e la sua collocazione topografica, poiché la pasticceria è sita in Via Padova e la pizzeria in Viale Ippocrate; lo stesso vale per altre tipologie commerciali, come Tecnocopia, nel medesimo viale Ippocrate, annunciato nel web come "Stampe a colori da $€ 0,15$ Tesi di laurea Cartucce Scansioni Piazza Bologna Roma - Tecnocopia". Analogamente, la mappa Google della zona riporta Vitaldent Roma Piazza Bologna in Via Sambucuccio d'Alando, Daruma Sushi Kosher Piazza Bologna in Viale delle Province e Compro Oro Roma Piazza Bologna in Via Lorenzo il Magnifico.

La prova più evidente del valore iperonimico dell'odonimo in questione è offerta dagli annunci di agenzie immobiliari; nel sito www.attico.it/roma/affitto/ appartamenti/ alla pagine della cosiddetta "zona Bologna" (accesso: 17 ottobre 2014) si leggono gli annunci per locali in Via Lorenzo il Magnifico, Via Matilde di Canossa, Via Catania, Viale Ippocrate, Via Michele di Lando, Circonvallazione Nomentana, Via Ravenna, Via Oreste Tommasini, Via Lucca, Via Lomellina, Via dei Canneti, Via De Mattheis, ecc., ossia aree di circolazione che, disposte su una carta, mostrano l'espansione notevole e topograficamente scarsamente giustificabile della "zona Bologna" indicata accanto a ciascun indirizzo. Analogamente, la descrizione locativa che di ogni appartamento offerto in affitto dà il sito www. mioaffitto.it alla voce "indirizzo" presenta, secondo uno schema di progressivo allargamento, la via dove sorge lo stabile, la zona, il quartiere, la città: per esempio "Enrico Stevenson no 24, Bologna, Nomentano, Roma", con il primo elemento variabile".

Altri esempi si ricavano dalla ricerca in rete delle voci congiunte "esercizi commerciali" "piazza Bologna Roma": il sito www.gaiaxroma.it (accesso: 17 ottobre 2014) restituisce 45 risultati, appartenenti a svariate categorie merceologiche presenti in aree di circolazione più o meno prossime alla piazza, comprese Via Rodolfo Lanciani, Via Cesare Rasponi, via Domenico Morichini e Via dei Fosca-

${ }^{6}$ Leggo nelle prime pagine gli odonimi Matilde di Canossa, delle Provincie, XXI Aprile, Livorno, Stamira, Alfonso Borelli, Padova, Berengario, Ravenna, Giuseppe Melchiorri, Salento, Arezzo, Apuania... 
ri. Altri ancora dall'indirizzo www.romatoday.it/aziende/zona/bologna dove alla voce "Imprese in zona Bologna" compaiono insegne relative a Viale Libia, Via dei Villini, Via di Villa Patrizi, Via Nomentana Nuova, Viale Regina Elena, Viale Regina Margherita, Via Giuseppe Antonio Guattani, Via Antonio Nibby, Viale del Policlinico, tutte piuttosto lontane da Piazza Bologna e comunque collocabili nel quadro di altre denominazioni iperodonimiche.

\section{Odonimi detoponimici della "zona Bologna"}

Tra gli odonimi sottintesi nell'iperonimo Bologna figurano ovviamente tutte le strade che si dipartono (o sboccano) nella piazza: Viale XXI Aprile, Via Livorno, Via Michele di Lando, Via Lorenzo il Magnifico, Viale delle Provincie, via Sambucuccio d'Alando e Via Ravenna. Due di questi odonimi rappresentano importanti città italiane, Livorno e Ravenna (area di circolazione, la seconda, completata da un Largo Ravenna). La zona intorno è caratterizzata da altri nomi di alcune fra le maggiori città italiane, le cui denominazioni furono imposte in maniera non omogenea né contemporanea tra il giugno 1912 (primo blocco numeroso) e il marzo 1954 (ultimo blocco consistente) (fonte: Ufficio di Toponomastica del Comune di Roma; ricerca in rete: www.comune.roma.it/servizi/SITOWPS/ricercaAreeCircolazione.do; accesso: 9 ottobre 2014), tanto che esiste, come detto, un Viale delle Provincie (dal 1948) che sfocia, al capo opposto di Piazza Bologna, nel Piazzale delle Provincie (dal 1930); si noti la grafia provincie anziché la più corretta e diffusa province?

Nell'area di Piazza Bologna si collocano anche le vie Agrigento, Arezzo, Bari, Belluno, Benevento, Caserta, Catania, Catanzaro, Chieti, Como, Cosenza, Cremona, Cuneo, Foggia, Forli, Imperia, Livorno, Lucca, Massa Carrara, Padova, Pavia, Pisa, Potenza, Reggio Calabria, Rovigo, Siena, Siracusa, Trapani, Treviso, Udine, Verona e le piazze Lecce, Salerno e Sassari (34 città-province); inoltre 13 odonimi corrispondenti a subregioni, le vie della Brianza e Lomellina (nella regione Lombardia), Carnia (in Friuli), Casentino, Chianti, Garfagnana e Lunigiana (in Toscana), Ciociaria (nel Lazio), Polesine (in Veneto), Capitanata e Salento (in Puglia), Marsica (in Abruzzo) e Ogliastra e la piazza del Campidano (in Sardegna), nonché 3 dedicati a comuni non capoluogo di provincia, le vie Pontedera (provincia di Pisa), Vigevano (provincia di Pavia) e Pontida (provincia di Bergamo, questa piazza per motivi storici legati alla lotta medievale dei Comu-

${ }^{7}$ Andrà precisato che in Italia, al contrario di numerosi altri Paesi, dal XX secolo le province (attualmente 110, anche se dal 2014 private di molti poteri e competenze amministrative e da più parti politiche se ne chiede l'abolizione come enti intermedi inutili e dispendiosi), salvo rare e recenti eccezioni, sono identificate dal toponimo capoluogo, motivo per il quale città (capoluogo) e provincia rappresentano un caso di piena omonimia (salvo l'uso normale della specificazione: provincia di Ravenna vs. città (comune, municipio) di Ravenna. 
ni contro il potere imperiale), un microtoponimo come Campaldino (comune di Poppi, provincia di Arezzo, in ricordo di una battaglia tra Guelfi e Ghibellini) e un toponimo non più esistente (Apuania, nome antico della città di Massa recuperato in epoca fascista per indicare l'insiene di Massa e di Carrara, presso le Alpi Apuane). A queste 52 aree di circolazione detoponimiche (ma nel comune di Roma, complessivamente, sono intitolate strade a circa due mila comuni italiani) occorre accostare, nella medesima zona, le vie dei Vespri Siciliani e della Lega Lombarda (contenenti un aggettivo decoronimico) e infine le vie Ruggero di Sicilia, Isabella d'Aragona, Eleonora d'Arborea, Matilde di Canossa, Giovanni da Procida, Contessa di Bertinoro, Alberto da Intimiano, Goffredo di Buglione e Sambucuccio d'Alando; in questi 9 casi il toponimo è parte integrante della catena antroponimica del personaggio celebrato nelle insegne e pertanto la filiera transonimica si arricchisce di un passaggio: toponimo (o coronimo nel caso di regione) $>$ antroponimo $>$ odonimo. Si tratta, in ogni caso, di un processo transonimico lineare con transonimie irrelate.

\section{Insegne, manifesti, depliant, vetrine come fonte di toponimi transonimizzati}

Tornando all'interno della stazione della metropolitana, il panorama onimico presenta manifesti e locandine di cinema, teatri e manifestazioni di vario genere; segnalo per es. il logo Cinecittà World, che contiene il microtoponimo della storica area di Roma dedicata alle produzioni cinematografiche; e la pubblicità del Sistina Story che, come titolo di spettacolo, è un ideonimo o pragmonimo (secondo le scelte terminologiche possibili) o comunque un nome proprio da ascrivere alla produzione dell'ingegno umano, la cui filiera risale al teatronimo Sistina, che prende il nome dall'odonimo deantroponimico (Via Sistina dal papa cinquecentesco Sisto V come pure il non più esistente Palazzo Sistino presso la Stazione Termini, mentre la Cappella Sistina in Vaticano prende il nome da un altro pontefice, Sisto IV).

Si osserva inoltre un distributore automatico di cibi e bevande, leggendo per es. Acqua di Nepi, Baci Perugina, Tramezzino Praga, Mozzarella e Pomodoro. Ma sui toponimi e gli aggettivi detoponimici nella gastronomia si tornerà oltre.

All'esterno della stazione (quattro gli accessi) s'impone nella pianta circolare della piazza l'edificio delle Poste Italiane completato nel 1935; a una porta a vetri del palazzo, un manifesto annuncia Romafil 2014, manifestazione filatelica. Roma, al pari di altri toponimi, si combina sempre più spesso con elementi onimici, anche in formule abbreviate o acronimi, per indicare qualcosa che si svolge nel proprio territorio.

I portoni degli stabili esibiscono i citofoni con i nomi degli inquilini; va ricordato che la piazza era agli inizi del XX secolo il centro di un'area destinata 
alla borghesia medio-alta, caratterizzata da villini a quattro piani con rifiniture architettoniche pregevoli, ma in epoca fascista (1922-1943) mutò il proprio carattere urbanistico con l'edificazione di grandi condomini, accresciuti ulteriormente con i grandi flussi immigratori dalla fine della Seconda guerra mondiale agli anni 70 del secolo scorso; la vicinanza con la "Sapienza" Università di Roma motiva le numerose presenze di giovani nei locali della zona e l'affitto di camere a studenti; per quanto negli anni gli appartamenti cambino evidentemente proprietari e inquilini, i cognomi detoponimici indicati rappresentano un indicatore attendibile dei movimenti demografici verso Roma e verso la zona Bologna. Si tratta di nomi di famiglia perlopiù meridionali: propongo l'unico esempio di Catanzariti, dall'etnonimo antico e grecofilo di Catanzaro (moderno: catanzarese), capoluogo di provincia in Calabria, anche per la casuale stretta vicinanza con Via Catanzaro, e nelle immediate vicinanze della piazza, i cognomi Cosentino e Napoletano, altri esempi di nomi di famiglia meridionali.

Gli stessi portoni presentano le cassette esterne per la cosiddetta posta condominiale, ossia i volantini pubblicitari. Fra i tanti, può capitare tra le mani quello di PizzaLampo, servizio a domicilio di pizze e altri piatti; segnalo, dai menù, le pizze Margherita Parmigiana, Margherita Amatriciana e Margherita Calabrese, le insalatone Mediterranea, Greca e Nicosia, la lasagna alla bolognese, ecc. Toponimi (ed etnici detoponimici) caratterizzano largamente la cucina italiana, indicando provenienza o specializzazione di certi tipici preparati. Limitando al mondo delle pizze, si possono incontrare nelle carte dei locali di tutta Italia, non meno di 4 mila differenti denominazioni [Caffarelli 2013b], buona parte delle quali appunto equivalenti a toponimi e ad etnici ${ }^{8}$. Tali denominazioni di pizze possono ripartirsi secondo cinque principali tipologie transonimiche: correlazione reale con la città o regione indicata (preparazione tipica del luogo); correlazione reale con l'area su cui insiste l'esercizio, indipendentemente da una specificità culinaria; correlazione metonimica (la farcitura della pizza contiene un ingrediente tipico del luogo, anche in mancanza di una tradizione pizzaiola: per es. Treviso per il radicchio, Bronte per il pistacchio, Pantelleria per i datteri, Alto Adige per lo speck, Tropea per la cipolla, Valle d'Aosta per la fontina, Calabria per il salame piccante, Belgio e Fiandre per l'indivia, Londra per il bacon, Caribe per frutti esotici, città e paesi nordeuropei per il salmone, toponimi cinesi per la soia, ecc.); evocazione generica di località con una forte e popolare tradizione pizzaiola (in particolare Napoli e la

${ }^{8}$ Per limitarsi alle voci inizianti con la lettera A-, ben oltre la cinquantina, segnalo le pizze: Abruzzo/-ese, Acapulco, Acitrezza, Acropoli, Adige, Adriatica, Afragolese, Africana, Agadir, Agerola/-ese, Agrigento/-ina, Alaska, Alassio, Alberobello, Albese, Alessandrina, Alghero, Alicudi, Alpi-ina, Alsaziana, Alto Adige/Altoatesina, Amalfi/itana, Amantea, Amatricel-iana, Ambrosiana, Amburgo, Americana, American Parmigiana, Anacapri, Anconetana, Angrisella, Appia Antica, Aquileia, Aragon, Arche Scaligere, Arezzo, Argentina, Arizona, Artica, Ascolana, Asiago, Asolana, Assergi, Atene, Atlantide, Austriaca, Avenza, Aversana; aggiungo, doverosamente, le pizze Bologna e Bolognese, voci perlopiù metonimiche per 'mortadella'. 
Campania) o con attrattività di carattere turistico, esotico, ecc.; infine, mancanza di qualsiasi correlazione (in questo caso i toponimi assumono un valore classificatorio, al pari di un qualsiasi numero, e sono fungibili tra loro, come nel caso di alcuni gruppi omogenei di nomi di luogo transonimizzati in odonimi).

Rimanendo in àmbito alimentare, i menù di ristoranti, trattorie, bar, pub, gelaterie e le vetrine di fornai, salumieri, ortolani, pasticceri presentano un'ampia gamma di toponimi crematonimizzati. Presso il Forno Bucarelli della vicina Via Stamira, sono esposti in prima fila i biscotti Napoletani e Ungheresi, bottigliette del liquore Marsala (dal comune siciliano) e la Grappa di Teroldego (forma italiana dal tedesco Tiroler Gold, dunque con l'aggettivo etnico dal toponimo Tirolo). Presso la gastronomia Mercuri pizza a taglio in Viale XXI Aprile, oltre a vari toponimi e aggettivi detoponimici usati per formaggi, salumi e pizze, richiamano l'attenzione, nelle vetrine, la Polenta Valsugana (valle del Trentino), le Bontà d'Altamura (marchio di pani e simili dal comune del Barese), la serie di infusi Pompadour (dalla marchesa de Pompadour il cui nome è assai usato in gastronomia: il nome proprio è un predicato nobiliare, dunque un microtoponimo antroponimizzato - ovvero un antroponimo demicrotoponimico - e qui divenuto marchionimo) e i biscotti Pavesini; il monobrand (per usare la terminologia anglofila prevalente) Pavesini presenta una complessa filiera transonimica bidirezionale (con almeno due tappe caratterizzate da antroponimi): antroponimo Papia (ma l'etimologia non è da tutti condivisa) $>$ toponimo Pavia (città della Lombardia) $>$ etnico pavese $>$ cognome Pavese/Pavesi $>$ marchio aziendale dolciario Pavesi (dall'imprenditore Mario) $>$ singolo prodotto Pavesini (biscotti).

\section{Transonimie legate ai veicoli di superficie}

In Via Lorenzo il Magnifico si trova un concessionario di motociclette. Non sono pochi i modelli motociclistici che recano il nome di un toponimo e ben più numerosi sono i modelli automobilistici che denominazioni detoponimiche e cioè da toponimi legati a corse automobilistiche (Le Mans, Monza, Vallelunga, Daytona, ecc.); da toponimi relativi a costruttori e aziende (Lagonda, Modena, Maranello, ecc.); da toponimi di località turistiche, mete di viaggi e indicatori di status (Capri, Malibù, Bel Air, Maratea, Murano, Montecarlo, ecc.); da toponimi generici e con motivazioni varie (anche politiche, storiche ed economiche: per es. Camargue, Cayenne, Yalta, Brescia, Montreal, ecc.). Incontro nel mio esercizio di "namewatching" una Nissan Qashqai (che riprende il nome di una confederazione di clan di lingua turca che risiedono nel sud dell'Iran, di tradizione pastori nomadi), una Volkswagen Tiguan (dall'etnico di un popolo indiano d'America, da cui anche il nome della città messicana di Tijuana, anche se la vettura viene popolarmente reinterpretata come "tigre" + "iguana) e una Opel Meriva (centro turistico nel Mozambico) (ma per un repertorio assai più ampio, benché certo non esaustivo, rinvio a Caffarelli 2014b). 
I taxi di Roma - un posteggio taxi è sito in Piazza Bologna - sono in buona parte - quelli appartenenti alle cooperative Autoradiotaxi Romana, Pronto Taxi, La Capitale e Tevere, rispettivamente con nomi di città italiane, nomi di città straniere, nomi di Stati, e fiumi italiani e stranieri: un centinaio di voci in tutto, ciascuna inserita in una sigla alfanumerica, con semplice valore identificativo e distintivo, senza ovviamente alcuna correlazione. Incontro per es. le vetture Novara 6, Monza 32, Marino 8, Napoli 40, appartenenti a società differenti.

I veicoli di passaggio o parcheggiati presentano ulteriori repertori propriali transonimizzati: si pensi agli adesivi sui finestrini delle automobili e sulla carrozzeria delle moto; fra i tagliandi assicurativi si leggono facilmente Assicurazioni Milano, Padana Assicurazioni, Italiana Assicurazioni, Zurich Connect); tra i concessionari dove i veicoli sono stati acquistati, Autocentro Balduina, Autocolosseo, Appia Nuova Car, ecc., da microtoponimi romani.

Si aggiungano i nomi commerciali sui camion; le grandi pubblicità sulle fiancate dei bus, le denominazioni dei capolinea dei mezzi pubblici annunciati nei display: in Piazza Bologna e nelle più immediate vicinanze fermano per es. le linee di superficie 168 (Farnesina), 309 (S. Maria del Soccorso), 61 (Washington), 542 (Verano), 310 (Vescovio), 21 (Conca d'Oro), che esibiscono nel display un odonimo detoponimico, oppure un agioeconimo e o un antroponimo (il Viale Washington è dedicato al primo presidente degli Stati Uniti George) che tuttavia da un toponimo ha origine.

Le insegne più consultate dai cittadini sono le tabelle che elencano le singole fermate dei percorsi dei mezzi pubblici di superficie. Può essere curioso notare come tessere del grande mosaico odonimico della capitale si riproducano in queste liste dove hanno perduto ormai, nella competenza della maggior parte dei parlanti, specie le giovani generazioni e gli stranieri, il loro originario significato. La frequentata linea 62 parte da Via di Torre Argentina; l'area della via e dell'omonimo largo è detta dai romani più semplicemente l'Argentina e rappresenta, nel cuore della capitale, un altro tipico iperonimo odonimico al pari di Bologna ${ }^{9}$.

La linea 61 contempla, tra le fermate, S. Paolo del Brasile, Veneto, Bari, Catanzaro, Ravenna, Bologna appunto, Livorno, Marsica, Massa Carrara: tutti toponimi odonimizzati a Roma, cui si aggiungono le fermate Barberini, Lanciani, Bergamini, Fiorentini (cognomi di uomini o famiglie illustri, rispettivamente dai toponimi Barberino Val d'Elsa in Toscana, Lanciano in Abruzzo, Bergamo in Lombardia e Firenze $)^{10}$.

${ }^{9}$ I romani tuttavia ignorano, per la stragrande maggioranza, che l'iter detoponimico della voce ha una curiosa origine: non dallo Stato sudamericano (e neppure da un aggettivo di lessico), ma da Argentoratum, nome latino di Strasburgo in Francia, da dove proveniva l'alto prelato che fece edificare la piccola torre: la quale fu detta in àmbito colto argentoratina, ma semplificata dal popolo in argentina.

${ }^{10}$ Merita soffermarsi su Veneto: la relazione è infatti con la nota Via Veneto, la cui denominazione anagrafica ufficiale è però Via Vittorio Veneto (dal comune trevigiano che fu teatro 
La linea 13 registra, tra le altre, le fermate $S$. Martino della Battaglia, Largo Somalia (qui, inaspettatamente con il denotato accanto al denotante), Valle Melaina e Monte Massico, ricordando i blocchi odonimici, rispettivamente, delle grandi battaglie risorgimentali, del cosiddetto quartiere africano, delle numerosissime valli e degli altrettanto numerosi oronimi che pure figurano nelle insegne delle aree di circolazione romane. La linea 2 mostra il complesso itinerario immaginario che i gruppi odonimici della città determinano con i percorsi dei mezzi pubblici: Ripa Teatina, Rivisondoli e Lanciano (tre comuni abruzzesi) seguiti da Efeso e da Europa.

Sulla Piazza Bologna insistono le agenzie della Banca Popolare di Novara, della BNL (Banca Nazionale del Lavoro) - Gruppo PNB Paribas e di Intesa Sanpaolo - Agenzia Roma Piazza Bologna. Il toponimo Novara è scoperto, come in genere per le banche popolari che si identificano con una città; il toponimo Parigi è invece mimetizzato nella sigla complessa di una multinazionale; nel terzo caso l'odonimo individua la localizzazione precisa dell'agenzia; s'incontra nelle vicinanze anche la Banca Popolare dell'Emilia Romagna. Le banche costituiscono un tipico àmbito di utilizzo di nomi di luogo: il richiamo all'area d'interesse della cassa di risparmio o della banca di credito cooperativo o di altro tipo d'istituto di credito si esprime con almeno tre differenti tipologie: a) il toponimo pieno o quasi: Crediumbria, Emil Banca (per Emilia), Friuladria, Bancasciano (di Asciano), Cariprato e Carifano (con CARI acronimo di Cassa di Risparmio), Banca Popolare di Ancona, di Bari, di Spoleto, Mantovabanca 1896 e Rovigobanca; anche nella variante antica - Mediolanum 'Milano', BancaApulia 'Puglia' - ecc.; b) il toponimo ridotto a una sillaba o poco più in un acronimo: CaRIM (Cassa di Risparmio di Rimini), Carilo (di Loreto), CarispaQ (della provincia dell'Aquila), CariGE (Genova e Imperia), Creverbanca (Credito Veronese), BIVERbianca (Biella e Vercelli) ecc.; c) l'etnonimo: a parte rari casi - Antonveneta - espresso con voce aulica o metonimica: Apuana per Massa e Carrara, Atestina per Este, Camuna per Esine (Brescia), Laudense per Lodi, Malatestiana per Rimini, Irpina per Avellino, Credito Aretuseo a Siracusa.

\section{Transonimie in altri settori merceologici}

Non vi è praticamente settore merceologico che non contempli toponimi transonimizzati: lo ricordano, se fosse necessario, il negozio di calzature $\mathrm{Pa}$ olo in Via Lorenzo il Magnifico (nel settore calzaturiero per esempio, alcuni

della battaglia decisiva in favore dell'Italia contro l'Austria a conclusione della Prima guerra mondiale); la forma accorciata Veneto è quella usata dai romani e dai gestori del servizio pubblico di trasporto, ma ha una sua giustificazione storica: perché in origine la via si chiamava era appunto Veneto (la regione) e solo dal 1919 assunse l'altro nome. 
cataloghi battezzano sandali da spiaggia scarpe o da barca con nomi di località marittime e calzature utili per trekking in luoghi desertici con toponimi africani e asiatici evocativi, e così via); la vicina farmacia Il Magnifico (con minore evidenza e perlopiù in acronimo: la -b- di Cibalgina è notoriamente l'iniziale di Basilea; parte dell'aggettivo detoponimico Galenica Senese si ritrova nei prodotti Algosenac, Carbosen, Iopasen e Lidosen; il nome aziendale Mediolanum, equivalente a Milano, in Medipo, ecc.); esposti nella già menzionata Feltrinelli Point si notano facilmente, tra le novità in àmbito narrativo: Congo di David Van Reybrouck e Cairo Automobil Club di 'Ala al-Aswani (Feltrinelli, cognome che origina dal toponimo veneto Feltre), I love shopping a Hollywood di Sophie Kinsell (Mondadori), Pietroburgo di Andrej Belyi (Adelphi), oltre a classici come Il conte di Montecristo di Alexandre Dumas (dall'isola dell'Arcipelago Toscano), ecc.

Certo di maggior interesse è notare le insegne detoponimiche degli esercizi commerciali. Detto di quelli che traggono il nome dalle aree di circolazione di pertinenza, mi limito a citare nella Via Catanzaro, a poca distanza l'uno dall'altro: Di Nepi e Macallè (abbigliamento), Calabria doc (alimentari), e Mizzica Tipico Catanese (bar). Ora, qui siamo di fronte a tre differenti tipologie di crematonimi detoponimici: 1) insegna deantroponimica (il cognome, d'origine ebraica, $D i$ Nepi); 2) insegna detoponimica correlata (Calabria e Catanese - e nel primo caso accompagnata, curiosamente, da una seconda insegna "mozzarella di bufala campana", che comporta una non indifferente dislocazione topografica); 3) insegna detoponimica irrelata (Macallè città del Tigrè legata ai ricordi delle colonie italiane in Africa orientale e i due locali così chiamati si collocano agli angoli della Via Capitanata, subregione della Puglia, con Via Catanzaro.

\section{Le insegne stradali}

Esistono a Roma tre tipi di insegne stradali (tipologia molto importante, considerato che i principali studi sui panorami onimici hanno centrato l'attenzione sulle insegne pubbliche bilingui, in chiave sociolinguistica e politica); ovviamente le insegne di Roma sono esclusivamente in italiano; si trovano su tipiche targhe di marmo apposte sulle mura degli edifici; sui rettangoli metallici relativamente recenti imposti dalle norme della Commissione Europea; e nei segnali indicanti le direzioni da percorrere per accedere ad una data area, o i divieti di accesso o i vicoli senza sbocco (ciechi). L'area oggetto di analisi non offre motivi di particolare interesse.

Né si rinvengono scritte abusive sui muri degli edifici, mentre nella stagione estiva è possibile cogliere anche i nomi stampati sugli abiti - specie magliette - dei passanti (frasi spiritose, loghi di associazioni, slogan, griffe delle marche). 


\section{Conclusioni}

Il panorama onimico offerto da questa piazza romana e dai suoi dintorni ha consentito di spaziare su numerose tipologie di transonimizzazione di toponimi, italiani e no. I nuovi àmbiti nei quali si rinvengono i toponimi tali e quali o con lievi modifiche o negli aggettivi detoponimici (etnici) sono in particolare l'odonimia (considerabile certo un settore della microtoponimia, ma con caratteristiche formali, relazionali, funzionali e motivazionali perlopiù distinte); la crematonimia in tutte le sue varie sfaccettature e l'antroponimia, con particolare riferimento ai cognomi.

Abbiamo potuto notare come molto spesso il processo transonimico non si limita a un unico passaggio, ma costituisce un percorso nel quale più tipologie possono filtrare il toponimo di partenza e talvolta una di queste tappe è costituita da una fase di lessicalizzazione (con successiva rionimizzazione).

Appare inoltre chiaro, dagli esempi riportati, che l'intraprendenza transonimica di un nome di luogo non dipende dalla natura (discussa e discutibile, beninteso) di micro- o macrotoponimo, ma, prevedibilmente, dal potere del referente in termini di popolarità, garantibilità, memorabilità, evocatività, $\mathrm{o}$, più banalmente, da un accostamento spaziale (come nei tanti casi di esercizio commerciale che trae l'insegna dall'area di circolazione su cui insiste).

Va da sé che questo genere di ricerca sul campo potrebbe essere sviluppata, da un lato, attraverso il confronto con altri "onymic landscape" in Italia e in altri Paesi; dall'altro lato, con interviste ai parlanti per es. sull'uso orale degli odonimi (con la valutazione di iperonimi e iponimi), sulla presenza di toponimi transonimici nell'idiomappa mentale, e sulle opinioni esprimibili a proposito del riuso di nomi correlati e irrelati, in particolare fra i crematonimi.

\section{Bibliografia}

Caffarelli Enzo, 2014a, recensione di G. Puzey, New research directions in toponomastics and linguistics landscapes, [in:] Guy Puzey (ed.), Toponomastics and linguistic landscapes/Toponymie et paysages linguistiques / Toponomastik und sprachliche Landschaften, "Onoma. Journal of the International Council of Onomastic Sciences" 2011, 46, "Rivista Italiana di Onomastica" XX/1, 237-238.

Caffarelli Enzo, 2014b, Per un approccio al labirinto onimico dei modelli automobilistici italiani e stranieri, "Rivista Italiana di Onomastica" XX/1, 481-354.

Caffarelli Enzo, 2013, Il (gustoso) repertorio delle denominazioni delle pizze in Italia, [in:] Olivin Felecan (ed.), Name and naming. Proceedings of the Second International Conference on Onomastics: Onoma-stics in Contenporary Public Space, Baia Mare, May 9-11, 2013, Cluj-Napola, 107-118.

Landry Rodrigue, Bourhis Richard Y., 1997, Linguistic Landscape and Ethnolinguistic Vitality. An Empirical Study, "Journal of Language and Social Psychology" 16/1, 23-49.

Lucantoni Federica, Porcaro Elisa, Vigliarolo Bruno, 2009, L'odonimia ufficiale e colloquiale di Tor 
Bella Monaca, [in:] Enzo Caffarelli, Paolo Poccetti (eds.), L'onomastica di Roma. Ventotto secoli di nomi. Atti del Convegno - Roma, 19-21 aprile 2007, Roma, 455-472.

Name and naming. Proceedings of the Second International Conference on Onomastics: Onomastics in Contemporary Public Space, Baia Mare, May 9-11, 2013, Cluj-Napoca, 107-118.

Nikitin Sergej, 2011, Dinamiche orientative nelle grandi città: toponimi d'uso e toponimi ufficiali a Roma, Monza e a Mosca, "Rivista Italiana di Onomastica" XVII/1, 67-80.

Poccetti Paolo, 2014, Microtoponimi e macrotoponimi nell'antichità, [in:] Artur Gałkowski, Renata Gliwa (eds.), Mikrotoponinia i makrotoponimia. Problematyka wstęnna/Microtoponymy and macrotoponymy. Preliminary problems, Łódź, 67-88.

Puzey Guy, 2011, New research directions in toponomastics and linguistics landscapes, [in:] Guy Puzey (ed.), Toponomastics and linguistic landscapes/Toponymie et paysages linguistiques / Toponomastik und sprachliche Landschaften, "Onoma. Journal of the International Council of Onomastic Sciences" 46, 211-226.

Room Adrian, 1996, An alphabetical guide to the language of name studies, Lanham, Maryland.

\title{
Enzo Caffarelli
}

\section{"Piazza Bologna" in Rome: case study of an onymic landscape. Macro- and microtoponyms in transonymic processes}

\author{
(Summary)
}

What is the end of a toponym is a question that may seem curious only to those who do not have full perception of the continuous recycling place names undergo, passing from one typology (or category) to another in the onymic ambit, or lexicalising themselves. This paper will try to: a) analyse a corpus of toponyms of every kind from a specific space and time ambit, that is a square in the city of Rome, Piazza Bologna, and its surroundings; b) examine the transonymic process such toponyms have undergone; c) verify whether there are recurrent transonymic paths or ones that are more specific for particular typologies of toponyms. The essay opens with an ample terminological introduction and is then articulated in chapters about hodonymic hyperonyms (as underground trains stations), hodonyms deriving from toponyms and transonymies linked to vehicles and various commodities sectors.

Słowa kluczowe: antroponim, chrematonim, leksykalizacja, hodonim, pejzaż onimiczny, włoska terminologia językoznawcza, toponim, transonimizacja.

Keywords: anthroponym, chrematonym, hodonym, lexicalization, name-watching, onymic landscape, toponym, Italian linguistic terminology, transonymization. 